\title{
Structure 0ptimization Design of Pulseoscillation Amplifier for Hydraulic Oscillator Based on Numerical Simulation
}

\author{
Yuqiang Xu, ${ }^{*}$ Bingshuo Li, Yating Li \\ School of Petroleum Engineering, China University of Petroleum (East China), China
}

\begin{abstract}
Hydraulic oscillator is one of the effective tools to solve the problem of high friction in directional drilling and horizontal drilling. However, there are some problems with this kind of tools such as high pressure loss and insufficient vibration force. Because of its self-excited oscillation characteristics, pulse oscillation amplifier can realize the amplification of pulse jet pressure under the condition of lowpressure loss, which is one of the effective ways to solve the above problems of hydraulic oscillator. In this paper, according to the working characteristics of hydraulic oscillator and the demand of pulse amplification, the structure of pulse oscillator amplifier was optimized based on numerical simulation method. Firstly, the geometric and numerical models of the pulse oscillator amplifier were constructed, and the flow field distribution and pulse amplification effect of the pulse oscillation amplifier under different structural parameters were simulated and analyzed, and the influence of different structural parameters on the pulse amplification effect was explored. Secondly, the structure of the pulse oscillator amplifier was optimized by Response Surface Method, and the optimal structure based on the effect of outlet pressure amplification was obtained: upper nozzle diameter D1=22mm, upper nozzle length L1 $=26 \mathrm{~mm}$, lower nozzle diameter D2 $=28 \mathrm{~mm}$, lower nozzle length $\mathrm{L} 2=28 \mathrm{~mm}$, cavity length $\mathrm{L}=58 \mathrm{~mm}$, cavity diameter d=80 $\mathrm{mm}$, angle $60^{\circ}$. Its pressure loss was $0.3 \mathrm{MPa}$ and outlet pressure peak value was 4.5MPa, which was 1.8 times of the inlet pressure peak value of $2.5 \mathrm{MPa}$. Finally, the minimum relative error between the experimental results and the numerical simulation results was $4 \%$, which has verified the credibility of the numerical simulation and structural optimization results.
\end{abstract}

Keywords: Pulse oscillation amplifier, Numerical simulation, Structure optimization design, Response surface methodology

\section{Introduction}

With the acceleration of unconventional oil and gas exploration and development process, the drilling of horizontal wells accounts for about $70 \%$ of the total number of drilling in the world, and the length of horizontal section is increasing year by year. ${ }^{1}$ In the process of long horizontal section drilling, with the increase of well deviation angle and open hole section length, problems such as increased friction, serious overburden pressure and reduced ROP are becoming increasingly prominent, which seriously affect the well quality and drilling cycle. ${ }^{2}$ By modulating the hydraulic pulse, the hydraulic oscillator uses the hydraulic energy to make the drill string vibrate axially, so as to reduce the friction during the horizontal section drilling, which is one of the effective means to solve the above problems..$^{3-5}$ But at present, the tool has the problems of high-pressure loss and insufficient vibration force. ${ }^{6}$ If a pulse oscillation amplifier ${ }^{7,8}$ was added to the hydraulic oscillator, the self-excited oscillation characteristics of the pulse oscillation amplifier can be used to amplify the pulse pressure without significantly increasing the overall pressure loss of the tool, thus improving the vibration force and reducing drag effect.At present, the research on the pulse oscillation amplifier mainly focuses on the self-excited oscillation mechanism ${ }^{9,10}$ and numerical simulation method. ${ }^{11-14}$ Scholars have preliminarily proved its self-excited oscillation mechanism. On this basis, the point vortex and vortex ring numerical simulation models are proposed, which can simulate the self-excited oscillation flow field under the actual working condi-
Quick Response Code:

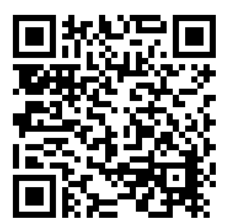

*Corresponding author: Yuqiang Xu, School of Petroleum Engineering, China University of Petroleum (East China), Qingdao, Shandong, 266580, China

Received: 23 January, 2021

Published: 26 February, 2021

Citation: Yuqiang Xu, Bingshuo Li, Yating Li. Structure Optimization Design of Pulseoscillation Amplifier for Hydraulic Oscillator Based on Numerical Simulation. Trends Petro Eng. 2021;1(1):1-9. DOI: 10.53902/TPE.2021.01.000503 
tions, and become an effective means to study the pulse oscillation amplifier. As the structure andsize of the pulse oscillator amplifier are the key factors affecting the effect of pulse amplification, the optimization design needs to be carried out comprehensively according to the working conditions, pulse characteristics and pressure amplification requirements of the hydraulic oscillator. Therefore, it is necessary to use the numerical simulation and design of experiment methodology to study the influence of structural parameters on the pulse amplification effect and pressure loss according to the working characteristics and pulse amplification requirements of the hydraulic oscillator, so as to optimize the structure of the pulse oscillation amplifier with low pressureloss and high pressurization effect.

\section{Materials and Methods}

\section{Establishment of geometric and mathematical model of pulse oscillator amplifier}

By analyzing the structure characteristics of the existing self-excited pulse oscillator amplifier, it is found that the self-excited pulse oscillation amplifier is axisymmetric, and the jet distribution is also symmetrical.Therefore, the self-excited pulse oscillator amplifier can be simplified as a two-dimensional problem on the X-Y plane. Figure 1 shows the two-dimensional geometric model and mesh generation of the self-excited pulse oscillator amplifier with the mesh number of 24199.

The flow mode of self-excited oscillation pulsed jet is turbulent. The methods of turbulence numerical simulation include Reynolds averaged simulation (RANS), direct simulation (DNS) and large eddy simulation (LES)14. Compared with the three simulation methods, it is difficult to get the details of the jet pulsation when the variables are homogenized in the Reynolds average numerical simulation. The direct simulation requires a high level of computer processing power, which is generally difficult to meet its requirements. However, LES is just between the direct simulation and the Reynolds average simulation, and can better describe the pulsation process of the pulsed jet. Therefore, the large eddy simulation method is used to simulate the self-excited oscillation pulse jet, and the geometric parameters of the self-excited oscillation cavity are optimized by taking the peak velocity of the jet as the monitoring index.

In the process of large eddy simulation, in order to simulate the large eddy flow field and establish the model of small eddy flow field, it is necessary to separate the large eddy flow field and the small eddy flow field through filtering processing. In large eddy simulation, the instantaneous flow variables are decomposed into large-scale and small-scale variables, which can be represented by weighted integrals in physical regions. After filtering, the velocity in the large eddy is decomposed into the sum of filtration velocity and its deviation, that is, the sum of large-scale component and sub grid scale component. The large eddy simulation solves the NSE(Navier Stokes equations) equations of large-scale flow field with variables.

The BOX filter function used in large eddy simulation is as follows:

$$
G\left|r-r^{\prime}\right|=\left\{\begin{array}{l}
\frac{1}{\Delta_{1} \Delta_{2} \Lambda_{3}},\left|r-r^{\prime}\right| \leq \frac{\Delta_{i}}{2} \\
0,\left|r-r^{\prime}\right|>\frac{\Delta_{i}}{2}
\end{array}\right\}
$$

By filtering the complete $\mathrm{N}-\mathrm{S}$ equation, the following results are obtained:

$$
\begin{gathered}
\frac{\partial u_{i}}{\partial \tau}+\frac{\partial\left(u_{i} u_{j}\right)}{\partial x_{j}}=\frac{1}{\rho} \frac{\partial p}{\partial x_{i}}+v \frac{\partial u_{i}}{\partial x_{i} \partial x_{j}} \\
\left\{\begin{array}{c}
\frac{\partial \overline{\mu_{i}}}{\partial \tau}+\overline{\mu_{j}} \frac{\partial \overline{\mu_{i}}}{\partial x_{j}}=-\frac{\partial p}{\partial x_{i}}+v \frac{\partial^{2} \overline{\mu_{l}}}{\partial x_{i} \partial x_{j}}+\frac{\partial \tau_{i j}}{\partial \partial x_{j}} \\
\frac{\partial \overline{\mu_{l}}}{\partial x_{i}}
\end{array}\right.
\end{gathered}
$$

The governing equations are as follows:

$$
\left\{\begin{array}{c}
\frac{\partial \mu_{i}}{\partial_{\tau}}+\overline{\mu_{j}} \frac{\partial \mu_{i}}{\partial x_{j}}=-\frac{\partial p}{\partial x_{i}}+\frac{1}{\operatorname{Re}} \frac{\partial^{2} \mu_{i}}{\partial x_{j} \partial x_{j}}+ \\
\frac{1}{\operatorname{Re}} \frac{\partial}{\partial x_{j}}\left(v_{\tau}\left(\frac{\partial \mu_{i}}{\partial x_{j}}+\frac{\partial \mu_{j}}{\partial x_{i}}\right)\right) \\
\frac{\partial \mu_{i}}{\partial x_{i}}=0
\end{array}\right.
$$

Where, $\boldsymbol{v}_{\boldsymbol{\tau}}=\left(\boldsymbol{C}_{\boldsymbol{s}} \boldsymbol{D}\right)^{\mathbf{2}}|\overline{\boldsymbol{S}}|$ modifies the eddy viscosity coefficient of smagorinshy model; D represents the attenuation function near the wall; and the smagorinshy constant $\mathrm{C}_{\mathrm{s}}=0.1$.

\section{Initial and boundary conditions}

The structure of the self-excited oscillation cavity is shown in Figure 1 . The inlet diameter is D1, the cavity diameter is D, the outlet diameter is D2, and the cone angle of the collision wall is $120^{\circ}$. Its working principle is that the pressure oscillation wave is generated by the reflection of the collision wall, and the oscillation cavitation is generated by the intense pressure oscillation of the fluid, and the large structure vortex is generated in the cavity with diameter $\mathrm{D}$ by the high-speed jet formed at the inlet D1. The cavitation effect of the self-excited oscillation cavity is enhanced by the interaction of the two vortices.

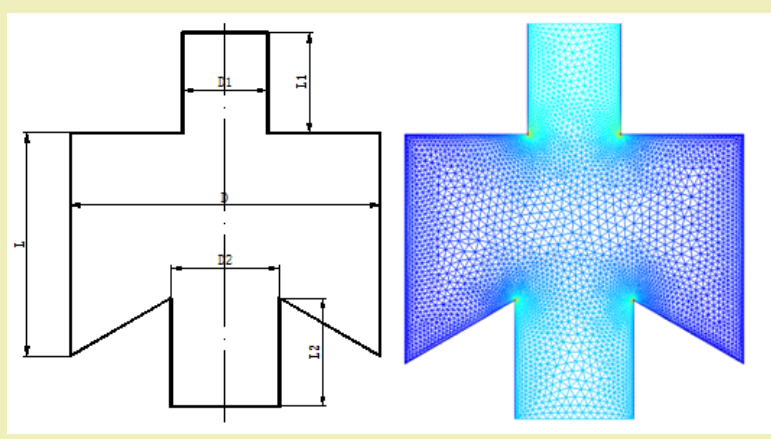

Figure 1: Two-dimensional geometric model and mesh generation.

As shown in Figure 2, the inlet pressure value of the pulse oscillation amplifier is calculated according to the parameters such as the eccentricity of the valve disc channel of the $\Phi 172$ hydraulic oscillator is $15 \mathrm{~mm}$, the eccentric distance of the eccentric flow channel is $30 \mathrm{~mm}$, the diameter of the eccentric flow channel is $\mathrm{d}=50 \mathrm{~mm}$, the flow rate is $32 \mathrm{~L} / \mathrm{s}$, and the valve disc speed is $1500-1800 \mathrm{r} / \mathrm{min}$, etc., as shown in Figure 3. 


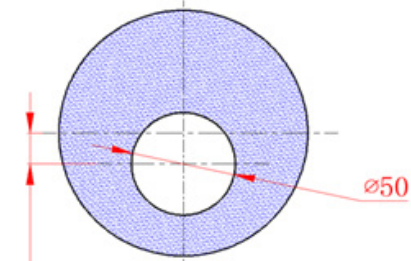

15 Complete coincidence/ $0^{\circ}$

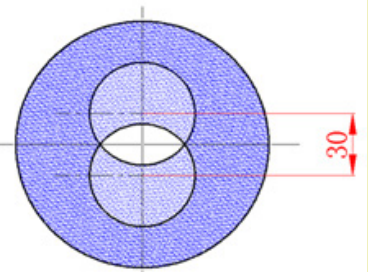

Maximum dislocation $180^{\circ}$
Figure 2: The working state of valve disc of hydraulic oscillator.

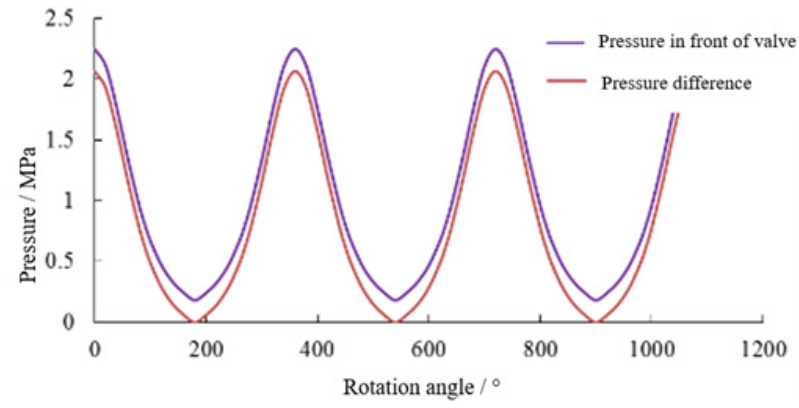

Figure 3: Inlet pressure of pulse oscillator amplifier.

Therefore, the boundary conditions are as follows: pressure inlet 2.5MPa (periodic change); flow outlet, viscosity is $0.062 \mathrm{~Pa} \bullet \mathrm{s}$, density is $1450 \mathrm{~kg} / \mathrm{m}^{3}$.

\section{Results and Discussion}

\section{Preliminary determination of structural parameters}

The optimal inlet diameter D1 of the self-excited oscillation cavity is calculated according to formula (1). When the pressure is $2.5 \mathrm{MPa}$ and the flow rate of the liquid pump is $25 \mathrm{~L} / \mathrm{s}$, the inlet diameter can be calculated by formula (5); according to the experimental and simulation results of relevant researchers, ${ }^{6-10,17}$ when D2/D1=1.2 1.4, L1/D1=0.4 0.7, D/D2=4 8, the pulse effect generated by self-excited oscillation is the best. Other dimensions are matched by structural parameters as shown in Table 1.

$$
D 1=5.3 \sqrt{\frac{q}{a \sqrt{p}}}
$$

Table 1: Design ofstructure parameters.

\begin{tabular}{|c|c|c|c|c|c|c|c|}
\hline NO. & \multirow{6}{*}{ D1 } & D2 & $\mathbf{L}$ & D & L1 & L2 & Angle $/{ }^{\circ}$ \\
\hline 1 & & $26 \mathrm{~mm}$ & $54 \mathrm{~mm}$ & $80 \mathrm{~mm}$ & 26 & 26 & 45 \\
\hline 2 & & $28 \mathrm{~mm}$ & $56 \mathrm{~mm}$ & $90 \mathrm{~mm}$ & 26 & 28 & 60 \\
\hline 3 & & $30 \mathrm{~mm}$ & $58 \mathrm{~mm}$ & 100 & 30 & 30 & 75 \\
\hline 4 & & $32 \mathrm{~mm}$ & $60 \mathrm{~mm}$ & 110 & -- & -- & 90 \\
\hline 5 & & $34 \mathrm{~mm}$ & $62 \mathrm{~mm}$ & 120 & -- & - - & - - \\
\hline
\end{tabular}

Where $\mathrm{q}$ is the jet flow rate, $\mathrm{L} / \mathrm{min}$; $\mathrm{a}$ is the flow coefficient (0.95); D1 is the inlet diameter of the self-excited oscillation chamber, $\mathrm{mm}$; $\mathrm{P}$ is the inlet pressure, MPa. Given that the flow rate is $25 \mathrm{~L} / \mathrm{s}$ and $\mathrm{P}$ is $2.5 \mathrm{MPa}, \mathrm{D} 1=22 \mathrm{~mm}$ is calculated by formula (5).

\section{Influence of structural parameters on pressure amplifi- cation effect}

On the basis of the preliminary design of the structure, based on the established geometric and mathematical model, the numerical simulation software COMSOLis used to simulate the pulse oscillation amplifier. The influence of the structural parameters of the pulse oscillation amplifier on the amplitude and pressure loss is revealed, and the structural parameters are optimized.

Firstly, the pressure amplitude amplification effect of throttling pulse is studied, and the influence of different structural parameters on it is analyzed. Take the outlet diameter as an example to introduce.The relevant structural parameters are shown in Table 2.

Table 2: Design ofstructure parameters.

\begin{tabular}{|c|c|c|c|c|c|c|c|}
\hline No. & \multirow{6}{*}{$\mathrm{D} 1=22 \mathrm{~mm}$} & D2 & $\mathbf{L}$ & D & L1 & L2 & Angle ${ }^{\circ}$ \\
\hline 1 & & $26 \mathrm{~mm}$ & $54 \mathrm{~mm}$ & $90 \mathrm{~mm}$ & $26 \mathrm{~mm}$ & $28 \mathrm{~mm}$ & 60 \\
\hline 2 & & $28 \mathrm{~mm}$ & & & & & \\
\hline 3 & & $30 \mathrm{~mm}$ & & & & & \\
\hline 4 & & $32 \mathrm{~mm}$ & & & & & \\
\hline 5 & & $34 \mathrm{~mm}$ & & & & & \\
\hline
\end{tabular}

Figures 4-13 show the variation of jet velocity and outlet pressure with time when cavity length $\mathrm{L}=54 \mathrm{~mm}$, cavity diameter $\mathrm{d}=90 \mathrm{~mm}$, lower nozzle diameter $\mathrm{D} 2=26 \mathrm{~mm}$ and initial pressure $\mathrm{P}=2.5 \mathrm{MPa}$.

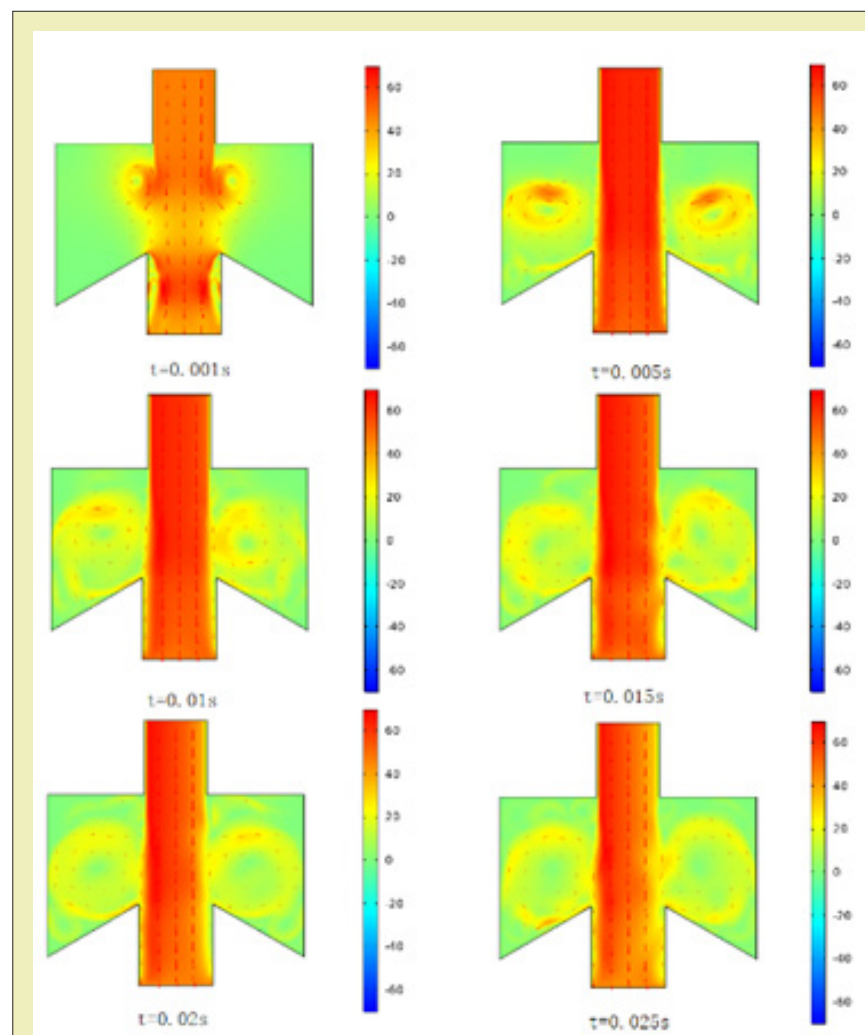

Figure 4: Velocity nephogram at different times when D2 $=26 \mathrm{~mm}$. 


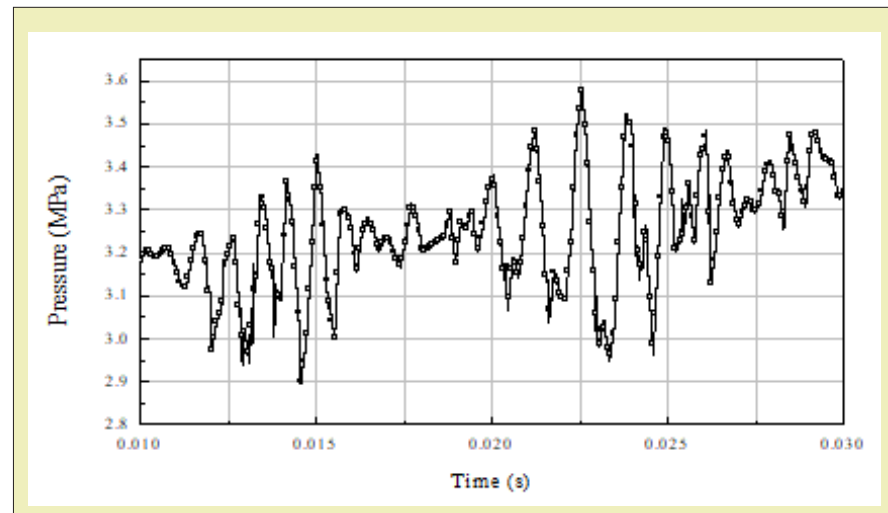

Figure 5: Change of outlet pressure when D2=26mm.

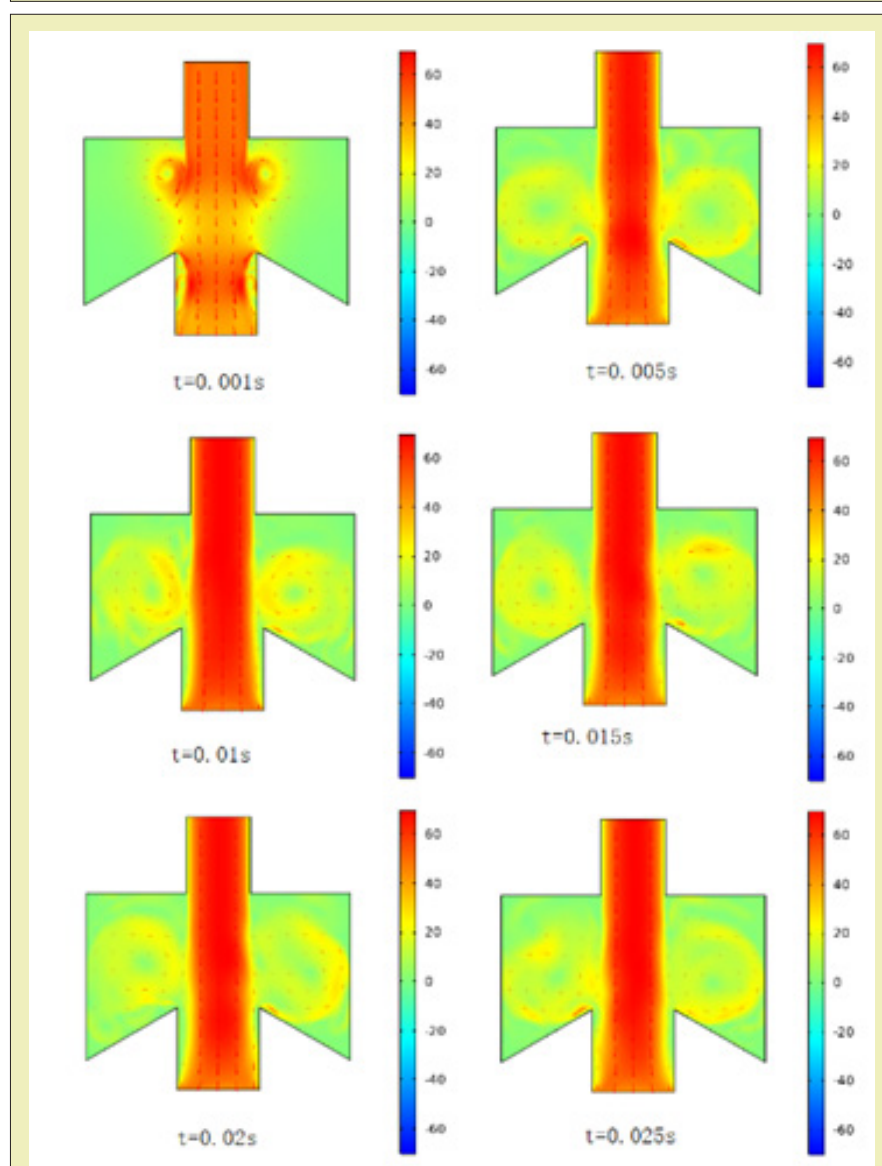

Figure 6: Velocity nephogram at different times when D2=28mm.

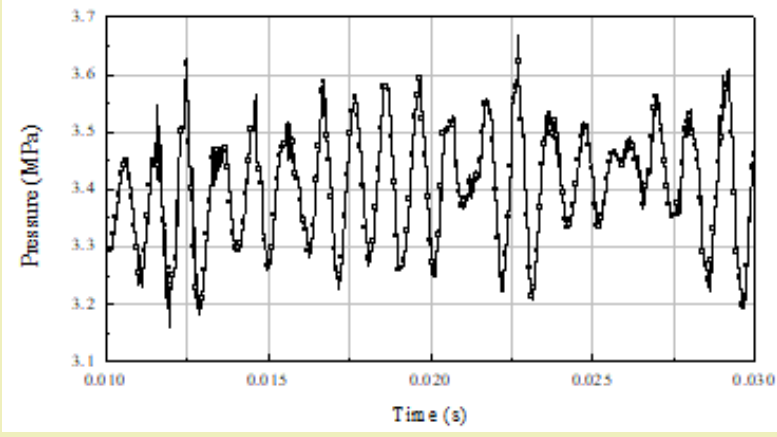

Figure 7: Change of outlet pressure when D2=28mm.
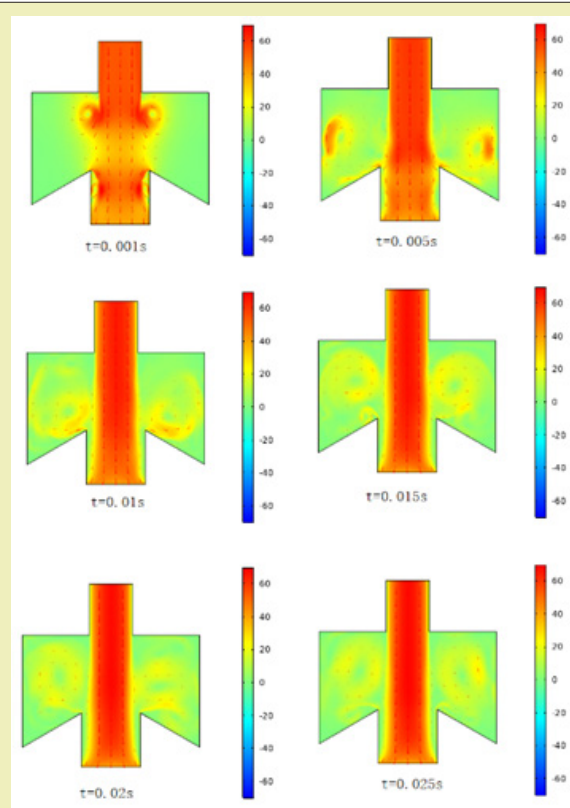

Figure 8: Velocity nephogram at different times when D2 $=30 \mathrm{~mm}$.

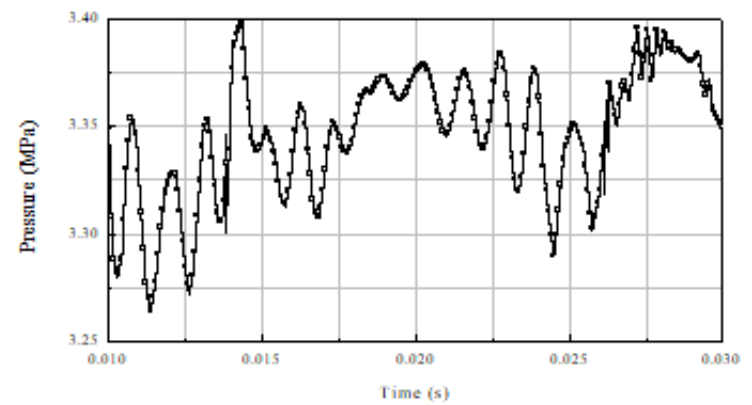

Figure 9: Change of outlet pressure when D2 $=30 \mathrm{~mm}$.
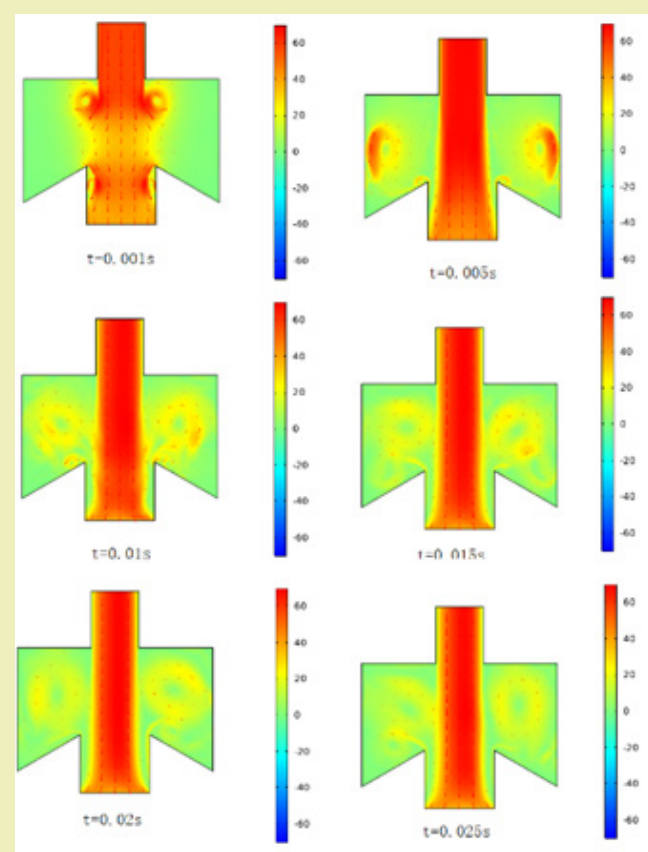

Figure 10: Velocity nephogram at different times when D2 $=32 \mathrm{~mm}$. 


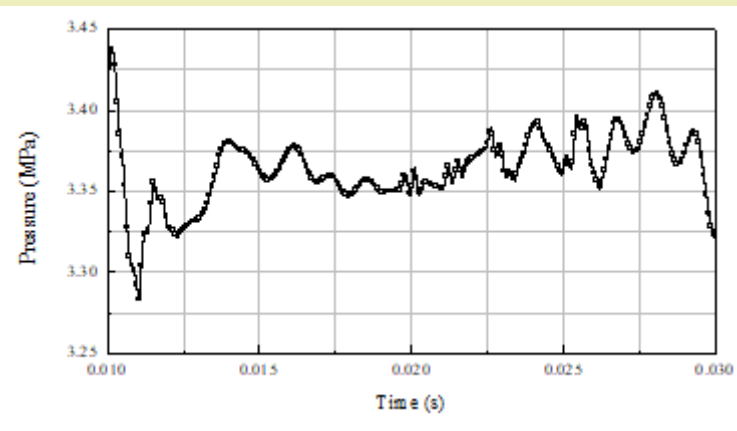

Figure 11: Change of outlet pressure when D2 $=32 \mathrm{~mm}$.

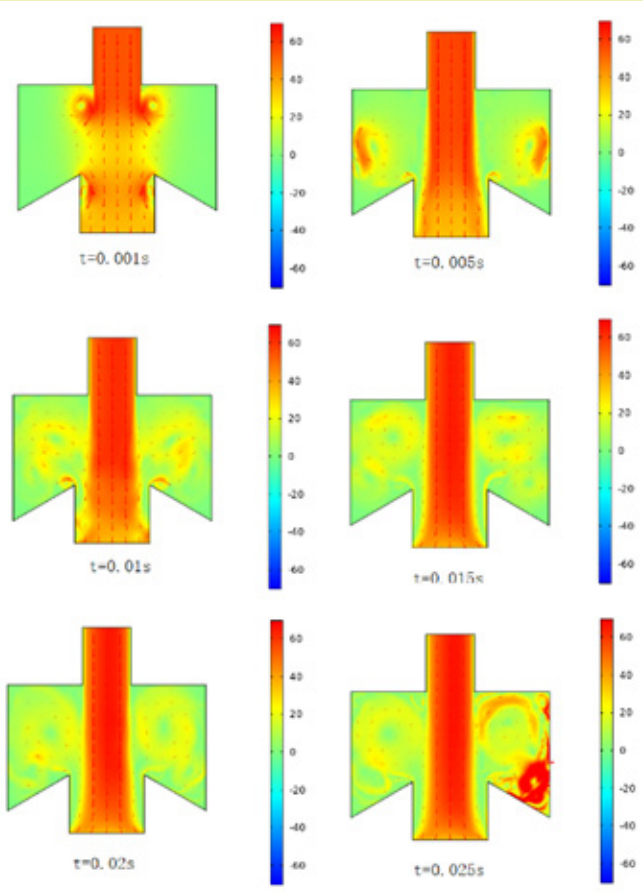

Figure 12: Velocity nephogram at different times when D2 $=34 \mathrm{~mm}$.

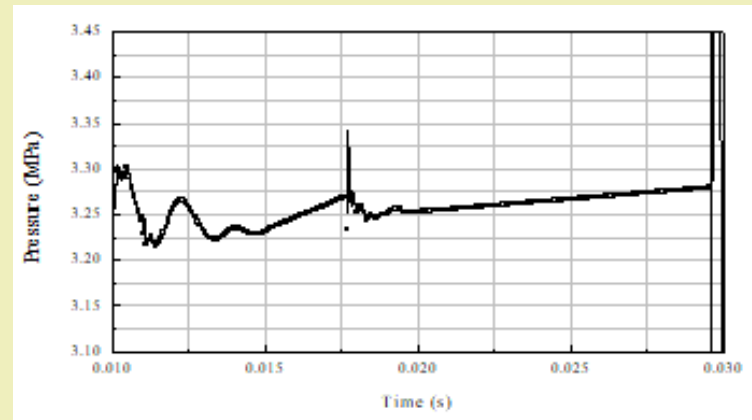

Figure 13: Change of outlet pressure when $D 2=34 \mathrm{~mm}$.

After processing the numerical simulation results, the curve of the peak value of the amplifier outlet pressure amplitude with D2 is drawn, as shown in Figure 14. When the inlet pressure is constant, the peak pressure of self-excited oscillation pulse first increases and then decreases. When D2 $=28 \mathrm{~mm}$, the peak pressure has a maximum value, and the effect of self-excited oscillation pulse jet is the most obvious. The reason for this phenomenon is that the diameter of the lower nozzle is too small, and the jet shrinks sharply when it enters the cylindrical pipe, resulting in a large energy loss, which affects the velocity of the jet flow out. When the diameter of the lower nozzle is too large, it can be seen from the generation principle of the self-excited oscillation pulse jet that the difference between the upper and lower nozzle diameters is too large to form vortex amplification at the outlet of the front nozzle, which leads to the poor effect of self-excited oscillation pulse jet.

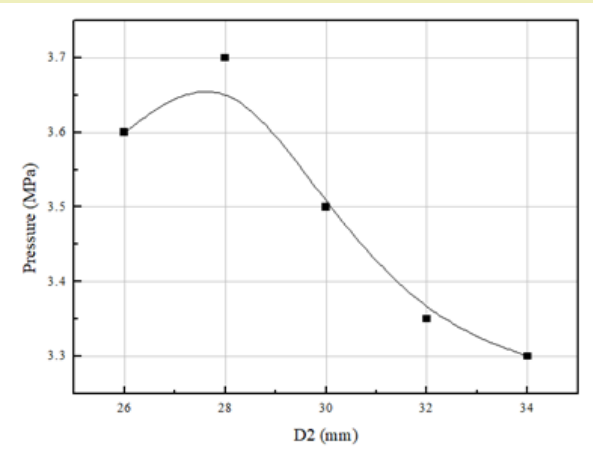

Figure 14: Variation of outlet pressure amplitude with outlet size D2.

Through the analysis of Figure 14 and the velocity nephogram under different D2 conditions, it can be seen that when D2 is $28 \mathrm{~mm}$, the throttling pulse pressure amplification effect is better, and the fluctuation form is more regular. Similarly, the effects of different cavity diameter, convergence angle, cavity length, upper nozzle length and lower nozzle length on pulse amplification effect are simulated, as shown in Figures 15-20.

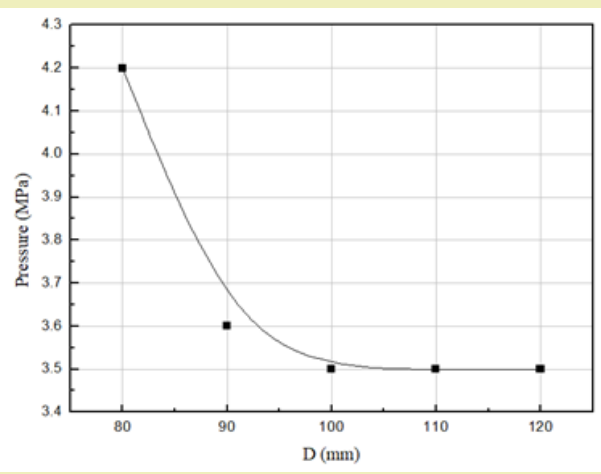

Figure 15: Variation of outlet pressure amplitude with cavity diameter D.

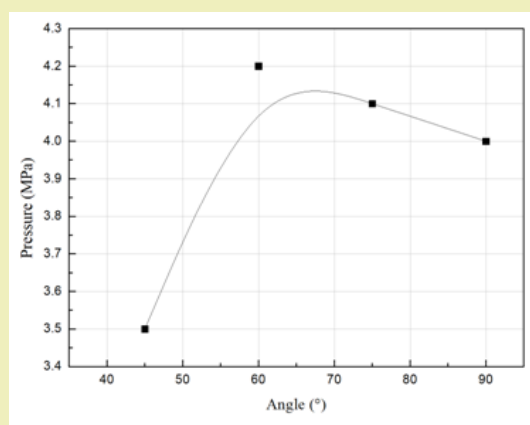

Figure 16: Variation of outlet pressure amplitude with angle. 


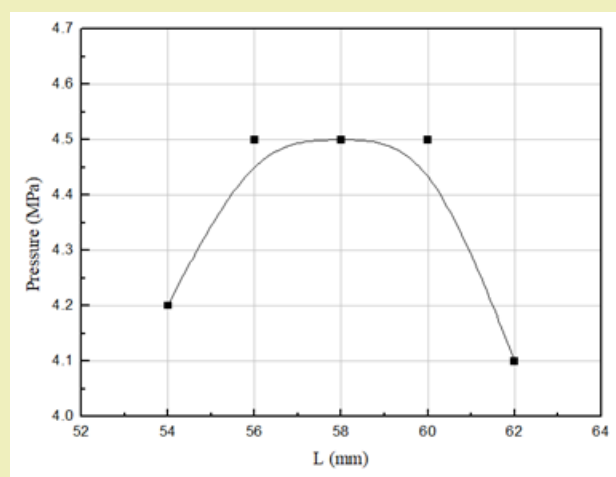

Figure 17: Variation of outlet pressure amplitude with L.

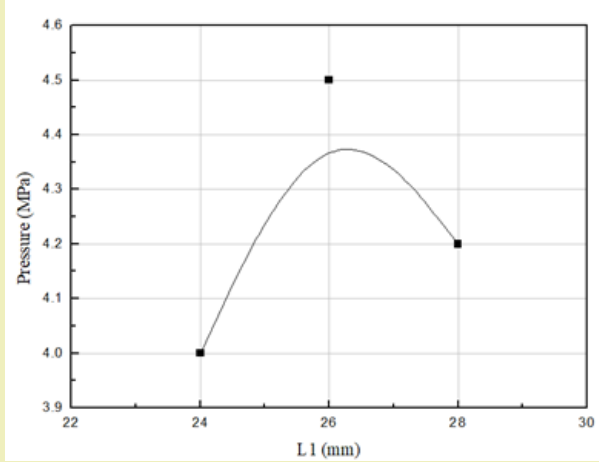

Figure 18: Variation of outlet pressure amplitude with L1.

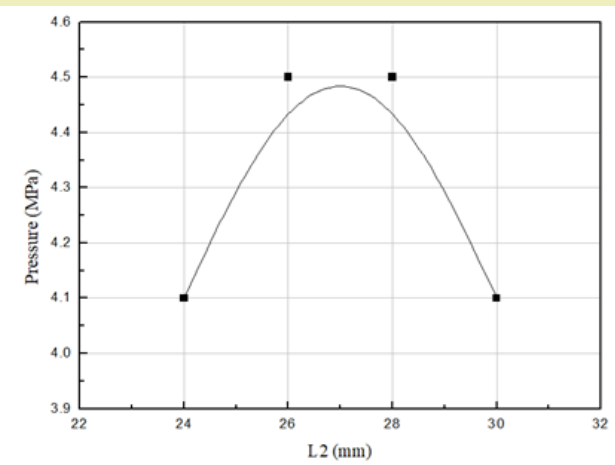

Figure 19: Variation of outlet pressure amplitude with L2.

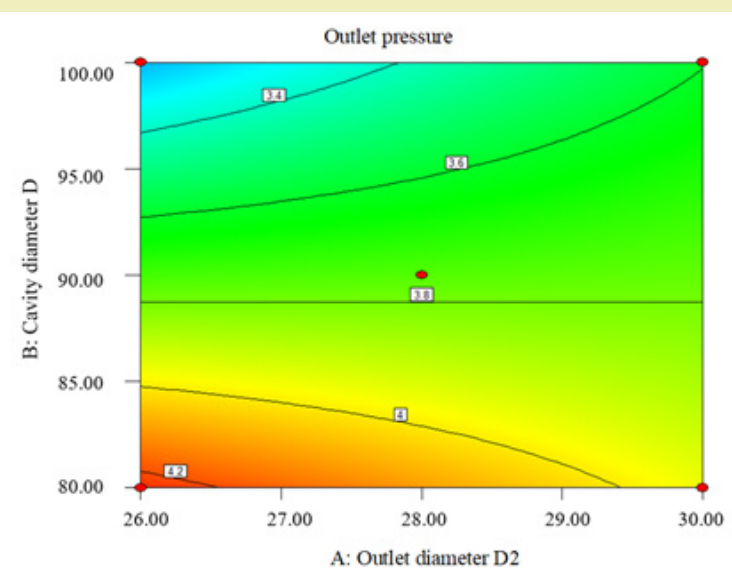

Figure 20: Response diagram of interaction between $\mathrm{D} 2$ and $\mathrm{D}$.
It can be seen that L1 and L2 have little effect on the effect of outlet pressure amplification, while the diameter of lower nozzle D2, the diameter of cavity D and the length of cavity $L$ have greater influence.

\section{Optimization design of structure parameters of pulse oscillator amplifier}

Through the previous study, the following optimal structural parameters can be obtained. That is: D1 $=22 \mathrm{~mm}, \mathrm{D} 2=28 \mathrm{~mm}$, $\mathrm{d}=80 \mathrm{~mm}, \mathrm{~L} 1=26 \mathrm{~mm}, \mathrm{~L} 2=26 \sim 28 \mathrm{~mm}, \mathrm{l}=58 \sim 60 \mathrm{~mm}$, angle $60 \sim 75$ degrees.

The Response Surface Method (RSM)15 is used to analyze the multi factors. Central composite design (CCD) and box Behnken matrix design (BBM) are the most commonly used experimental design methods of RSM. Since BBM is suitable for 2 5 factors optimization experiments, this paper uses BBM to establish response surface optimization model. The experimental design includes three factors: D2, D and l, which have three levels respectively. The experimental design scheme and response value design factor level values are shown in Table 3.

Table 3: Experimental design and response values.

\begin{tabular}{|c|c|c|c|c|}
\hline \multirow{2}{*}{ NO. } & \multicolumn{3}{|c|}{ Design variable parameters } & \multirow{2}{*}{$\begin{array}{c}\text { Pressure/ } \\
\text { MPa }\end{array}$} \\
\hline & $\mathrm{D} 2 / \mathrm{mm}$ & $\mathrm{D} / \mathrm{mm}$ & $\mathrm{L} / \mathrm{mm}$ & \\
\hline 1 & 28 & 90 & 58 & 4.1 \\
\hline 2 & 28 & 80 & 54 & 4.1 \\
\hline 3 & 30 & 80 & 58 & 3.9 \\
\hline 4 & 28 & 100 & 54 & 3.52 \\
\hline 5 & 30 & 90 & 54 & 3.4 \\
\hline 6 & 26 & 90 & 62 & 4 \\
\hline 7 & 30 & 100 & 58 & 3.34 \\
\hline 8 & 30 & 90 & 62 & 4 \\
\hline 9 & 26 & 100 & 58 & 3 \\
\hline 10 & 28 & 80 & 62 & 4 \\
\hline 11 & 28 & 100 & 62 & 3.8 \\
\hline 12 & 26 & 90 & 54 & 3.28 \\
\hline 13 & 26 & 80 & 58 & 4.2 \\
\hline
\end{tabular}

Figures $21 \& 22$ show the interaction of structural parameters on outlet pressure, and each line in the figure represents a gradient value of pressure. It can be seen from the figure that when $D$ is less than $85 \mathrm{~mm}$, the larger D2 is, the smaller the outlet pressure is, indicating that there is an optimal value. It can be seen from Figure 21 that when 1 is at a lower level, the outlet pressure decreases with the increase of D2, and when l is greater than $58 \mathrm{~mm}$, the outlet pressure increases with the increase of D2. It can be seen from Figure 20 that the outlet pressure increases with the increase of D regardless of the cavity length. When D1 $=22 \mathrm{~mm}$, D2 should be greater than $28 \mathrm{~mm}$, the optimal value of $\mathrm{L}$ is $58-62 \mathrm{~mm}$, and the optimal value of $\mathrm{D}$ is greater than $80 \mathrm{~mm} \cdot{ }^{16-18}$ 


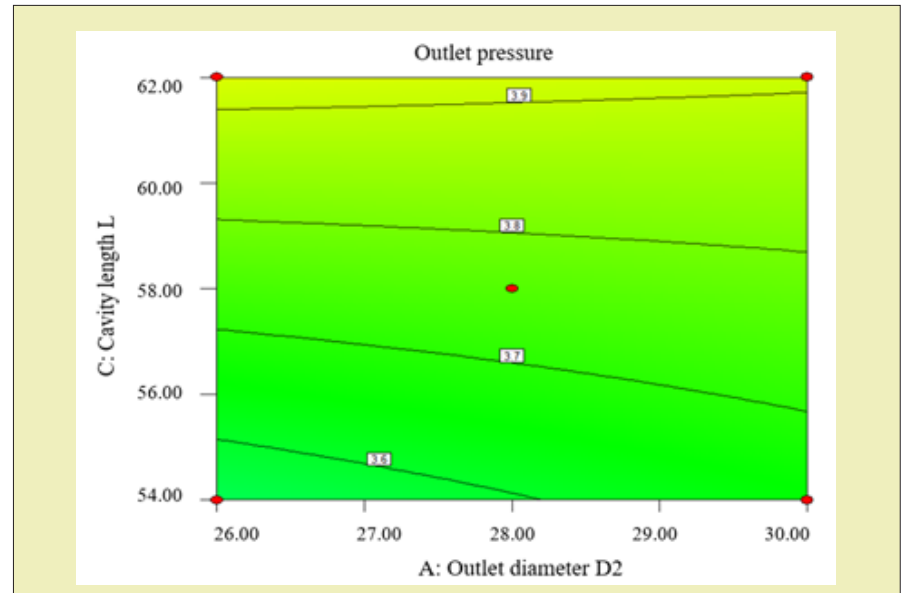

Figure 21: Response diagram of interaction between $\mathrm{D} 2$ and $\mathrm{L}$.

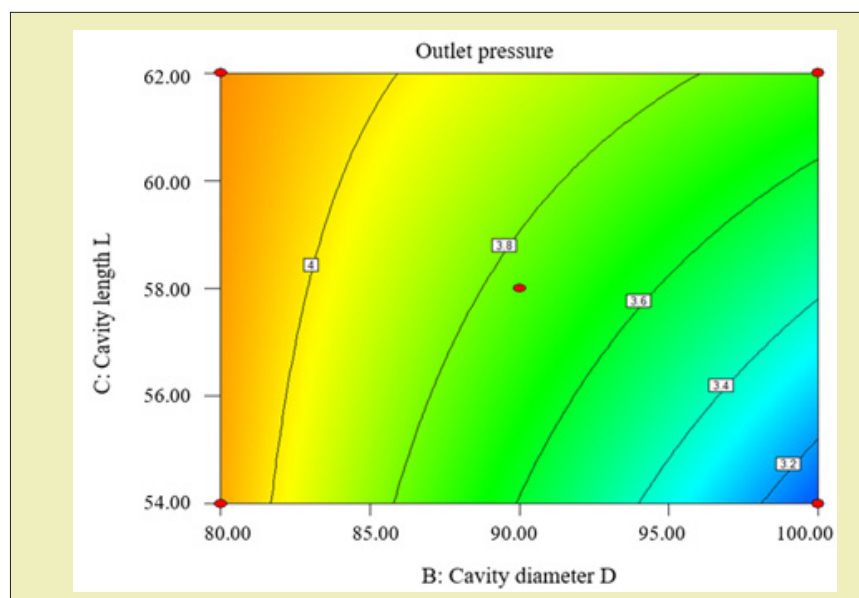

Figure 22: Response diagram of interaction between $\mathrm{D}$ and $\mathrm{L}$.

Using design expert software to analyze the design scheme and response value, the relationship between response value and structural parameters can be obtained.

\section{$\mathrm{P}=37.2-0.49 \mathrm{D} 2-0.47 \mathrm{D}-0.18 \mathrm{~L}+0.008 \mathrm{D} 2 \mathrm{D}-0.0038 \mathrm{D} 2 \mathrm{~L}+0.0036 \mathrm{DL}(6)$}

Figure 23 shows the internal student residual diagram of the outlet pressure. It can be seen from the figure that the residual points are well distributed near the straight line, which further indicates that the established mathematical model has high reliability.

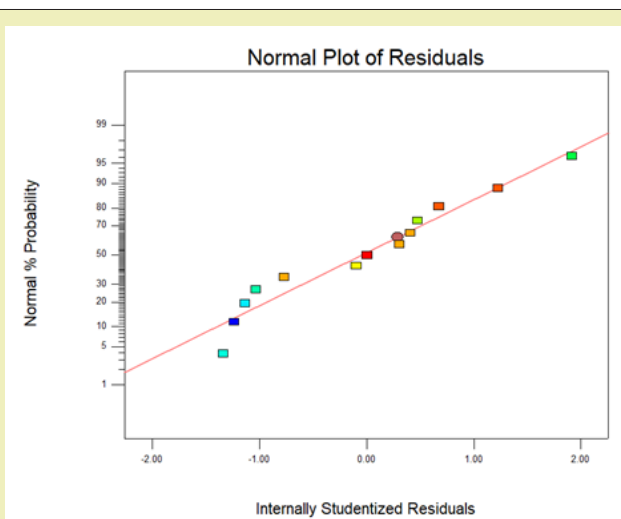

Figure 23: Internally studentized residual value of outlet pressure.
So far, the optimal structure of pulse oscillator amplifier based on the effect of outlet pressure amplification can be determined as follows:

The upper nozzle diameter D1=22 $\mathrm{mm}$, the upper nozzle length $\mathrm{L} 1=26 \mathrm{~mm}$, the lower nozzle diameter D2 $=28 \mathrm{~mm}$, the lower nozzle length $\mathrm{L} 2=28 \mathrm{~mm}$, the cavity length $\mathrm{L}=58 \mathrm{~mm}$, the cavity diameter $\mathrm{d}=80 \mathrm{~mm}$, and the angle is $60^{\circ}$.

Using the numerical method established above, the optimal structure is simulated and calculated. The peak pressure at the outlet is $4.5 \mathrm{MPa}$, which is 1.8 times of the inlet pressure peak value of $2.5 \mathrm{MPa}$, and the pressure loss is $0.3 \mathrm{MPa}$.

\section{Experimental verification}

\section{Construction of experimental device}

According to the test requirements, the experimental device is constructed, as shown in Figures 24\&25. Experimental equipment mainly includes: high pressure pump, fixed bracket, experimental tank, pressure gauge, etc. Among them, the rated displacement of high-pressure pump is $120 \mathrm{~L} / \mathrm{min}$.

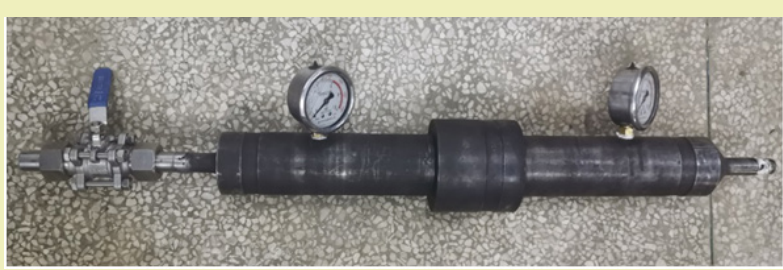

Figure 24: Experimental prototype.

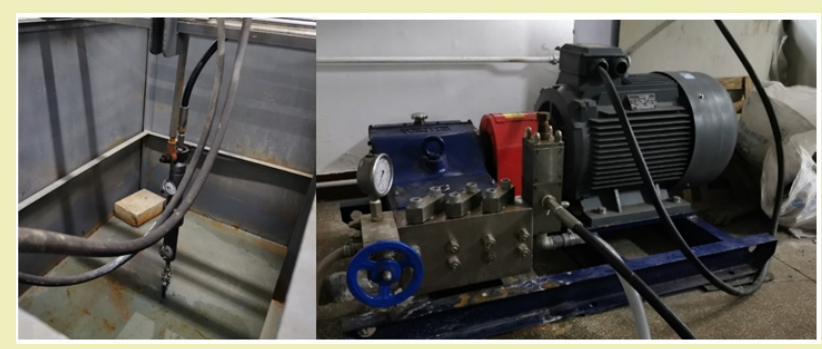

Figure 25: Experimental device.

\section{Experimental principle}

Because the rated displacement of the indoor test device is $120 \mathrm{~L} / \mathrm{min}$, it is difficult to realize the simulation condition of the actual working flow of $30 \mathrm{~L} / \mathrm{s}$. according to the Euler similarity principle, the actual resonance effect of the prototype can be evaluated by using the pressurization effect in the low displacement range (experimental conditions), and the accuracy of the numerical simulation results is verified.

According to the Euler similarity principle, the pressure $\mathrm{P}_{\mathrm{e}}$ and displacement $Q_{e}$ in the experiment should be equal to the pressure $\mathrm{P}_{\mathrm{n}}$ and displacement $\mathrm{Q}_{\mathrm{n}}$ in the numerical simulation.

$$
\frac{P_{s}}{\rho Q_{a}^{2}}=\frac{P_{n}}{\rho Q_{n}^{2}}
$$


It can be seen from the above formula that since the geometric dimensions, fluid density and displacement in the experimental and numerical simulation are consistent, the pressure of the two meets the similarity criterion, and the similarity ratio is 1 .

Therefore, under the condition of the same discharge capacity, the outlet pressure obtained by experimental test and numerical simulation meets the similarity criterion, and the similarity ratio is 1 , which can be directly compared and analyzed.

\section{Experimental scheme}

The outlet pressure of the prototype with different displacement of 55 90L/min was tested, and the outlet pressure and pressurization effect under the same conditions were simulated and analyzed by the established numerical simulation method. The numerical model was verified by comparing the experimental and numerical simulation results. It further confirms the credibility of the optimal structure and pressurization effect of the pulse oscillator amplifier optimized by numerical simulation.

As shown in Figure 26, under the condition of 55 90L/min displacement, the experimental test outlet pressure results and numerical simulation results have the same rule, that is, with the increase of displacement, the outlet pressure increases exponentially, and the gap between the experimental results and the simulation results gradually decreases.

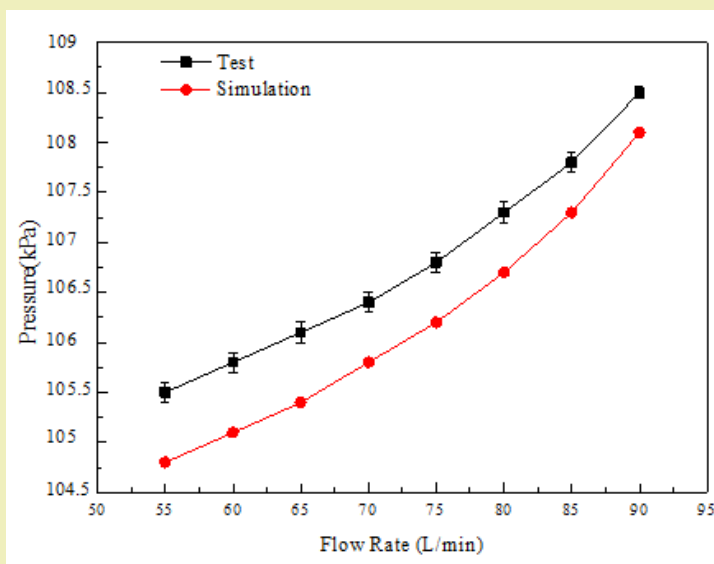

Figure 26: Comparison of experimental data and numerical simulation results.

Through calculation, the relative error between experimental and simulation results is reduced from $12 \%$ to $4 \%$ after the displacement is increased from $55 \mathrm{~L} / \mathrm{min}$ to $90 \mathrm{~L} / \mathrm{min}$. it can be seen that with the increase of displacement, the experimental results are more and more close to the numerical simulation results, which verifies the credibility of the optimal structure and pressurization effect of pulse oscillator amplifier optimized by numerical simulation in this project.

\section{Conclusion}

According to the working characteristics and pulse amplification requirements of $\Phi 172$ hydraulic oscillator, the geometric model, numerical model and boundary conditions of pulse oscillator amplifier are constructed. The flow field distribution and pulse amplification effect of pulse oscillation amplifier under different structural parameters are simulated and analyzed, and the influence law of different structural parameters on pulse amplification effect is explored.

The response surface method is used to optimize the structure of the pulse oscillator amplifier, and the optimal structure based on the effect of outlet pressure amplification is obtained: the upper nozzle diameter D1 $=22 \mathrm{~mm}$, the upper nozzle length $\mathrm{L} 1=26 \mathrm{~mm}$, the lower nozzle diameter D2 $=28 \mathrm{~mm}$, the lower nozzle length $\mathrm{L} 2=28 \mathrm{~mm}$, the cavity length $\mathrm{L}=58 \mathrm{~mm}$, the cavity diameter $\mathrm{d}=80 \mathrm{~mm}$, the angle is $60^{\circ}$; the pressure loss is $0.3 \mathrm{MPa}$, the outlet pressure peak is $4.5 \mathrm{MPa}$, which is the inlet pressure peak 8 times of $2.5 \mathrm{MPa}$.

The minimum relative error between the experimental results and the numerical simulation results is $4 \%$, which verifies the credibility of the numerical simulation and structural optimization results.

\section{Acknowledgments}

The authors would like to acknowledge the academic and technical supports of China University of Petroleum (East China).

\section{Funding}

None.

\section{Conflicts of Interest}

None.

\section{References}

1. Huachao S, Chunya LI, Zeying T, et al. Determining Optimum Length of Horizontal Section in Horizontal-well Development: Examples from Cn Gas Reservoirs, Country A. Natural Gas Technology and Economy. 2017.

2. Qingyou L, Jun J, Xiaohua Z. Control of friction resistance in drilling of horizontal well with long horizontal section. Oil Drilling \& Production Technology. 2016.

3. Chongdong S, Xinjiang C. Application of the Hydraulic Oscillator in Well 36-8-18H of the Sulige Block. China Petroleum Machinery. 2012.

4. Liu Z, Li R. Application of Hydraulic Oscillator with $\varphi 172 \mathrm{~mm}$ to Mediumto-shallow Horizontal Wells, Western Sichuan Basin. Natural Gas Technology and Economy. 2012.

5. Ruiqing M, Shizhong Z, Haitao W, et al. Research Status and Prospect of Hydraulic Oscillator Worldwide. Petroleum Drilling Techniques. 2015;43(5):116-122.

6. Hui Z, Zhonghua W, Wenjun C. Development and Field Testing of Hydraulic Oscillator. China Petroleum Machinery. 2014.

7. Yu Xiufang. Study on the strike characteristics of jet low-pressure highflow self-excited pulse cavitation. Shandong University. 2016.

8. Liu Guolai. The oscillation pulsed water jet pressure characteristics and self-sustained nozzle structure design research based on CFD. Beijing University of Technology. 2014.

9. Li Q Sha W, Li FB, et al. Oscillation Mechanism Analysis and Numerical Simulation for Tandem Self-Excited Oscillation Pulsed Jet Nozzle. 2014;448-453:3449-3453.

10. Tang Chuan-Lin, Hu Dong, Pei Jiang-Hong. Experimental study on the frequency characteristic of the self-excited oscillation pulsed nozzle. Acta PetroleiSinica. 2007;28(4):122-125.

11. Sagaut P. Large eddy simulation for incompressible flows: An introduction

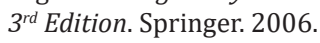


12. Verzicco FL, Orlandi P. Large-eddy simulation of flow around cylinder arrays at a subcritical Reynolds noumber. Journal of Hydrodynamics. 2008;20(4):403-413.

13. Yuan LL, Street RL, Ferziger JH. Large-eddy simulations of a round jet in crossflow. J Fluid Mech .1999;379:71-104.

14. Schluter JU, Schonfeld T. LES of jets in crossflow and its application to a gas turbine burner. Flow Turbulence Combust. 2000;65(2):177-203.

15. Kim SH, Na SW. Response surface method using vector projected sampling points. Structural Safety. 1997;19(1):3
16. Xu Y, Zhang H, Guan Z. Dynamic Characteristics of Downhole Bit Load and Analysis of Conversion Efficiency of Drill String Vibration Energy. Energies. 2021;14(1):229.

17. Xue L, Han H, Wang D, et al. Experimental study on jet pulse assembly design and numerical simulation. Pet Sci. 2020;17:222-231.

18. Zhang X, Peng J, Liu H, et al. Performance Analysis of a Fluidic Axial Oscillation Tool for Friction Reduction with the Absence of a Throttling Plate. Appl Sci. 2017;7(4):360. 